\title{
Preliminary Research on Modelling and Control of Two Line Kites for Power Generation
}

\author{
Ashley Yan \\ Electrical Engineering Department \\ Unitec Institute of Technology \\ Private Bag 92025, Victoria Street \\ West, Auckland 1142, New Zealand. \\ hyan@unitec.ac.nz
}

\author{
Nigel Yee \\ Electrical Engineering Department \\ Unitec Institute of Technology \\ Private Bag 92025, Victoria Street \\ West, Auckland 1142, New Zealand. \\ nyee@unitec.ac.nz
}

\author{
Loulin Huang \\ Mechanical \& Production \\ Engineering Department \\ Auckland University of Technology \\ School of Engineering, Computer \\ and Mathematical Sciences \\ Private Bag 92006, Auckland 1142, \\ New Zealand. \\ Loulin.huang@aut.ac.nz
}

\begin{abstract}
Wind power is the second largest renewable resource from which we can obtain electricity besides hydropower. Wind farms have had significant growth in past decades; however, they require a massive investment to setup including heavy towers, large land scale and huge blades. Betz's law states that the maximum power of the wind turbine can harvest only $59.3 \%$. Therefore, surfing kites have been proposed as a promising electricity generation alternative. This study aims to build a suitable simulation platform to study the modeling and control of kite for power generation.
\end{abstract}

In this paper, different types of the kite and their control paradigm have been reviewed and two-line surf kites have been identified as the subject of the investigation. By analyzing Newtonian mechanics theory, the dynamic model of a kite is established. Moreover, the kite power generation cycle and retraction cycle is explained.

In this paper, the simulation results demonstrate the power generation ability of a two-line surf kite. Considering the input constraints, a linear control system is proposed and simulated to make the kite achieve a figure eight configuration needed for power generation. The Simulink toolbox has then been employed to simulate the linear control scheme to determine its stability and performance.

Keywords - Wind power; Two-line Kite; Renewable energy;

\section{INTRODUCTION}

Since the innovation of electricity, its use in AC and DC motors and its application to something as essential as an electrical light bulb, human society's reliance on and consumption of electricity has grown at a steady rate from 7,323 billion watt-hours in 1980 to 19,710 billion watt-hours in 2012 [1]. This means electricity consumption globally is increasing at a rate of approximately $2 \%$ per annum. However, conventional power generation technologies can no longer cope with this growth. Meanwhile, there has also been concern about the reliance on fossil fuel-based electricity generation because of its impact on the environment and thus renewable technologies have become the favored method for power generation.
Wind generation through the use of wind turbines was fully developed on a mass scale in 1941 with the commissioning of the Smith-Putnam wind turbine in Castleton, Vermont, but the disadvantage of a wind turbine was immediately apparent as it was only operational for 1100 hours in total before a failure on the wind turbine blade occurred.

On the other hand, kite energy harvest is a new field in the renewable energy generation realm. Since it has been proposed, it has been quickly accepted by the public due to significantly fewer materials and weight advantages compared with the conventional wind turbines. It can offer a much cheaper option of utilizing wind power. Meanwhile, a kite can reach much higher altitudes than wind turbines where the wind speed, density, and stability are more suitable for electricity generation. This suggests that a kite will collect more energy and produce more steady output. Furthermore, according to the Betz' limitation, no turbine can capture more than $59 \%$ of wind kinetic energy on the horizontal axis. However, the high altitude kite is not subject to this limitation. [2] Figure 1 shows the wind speed and wind density at different elevation levels:

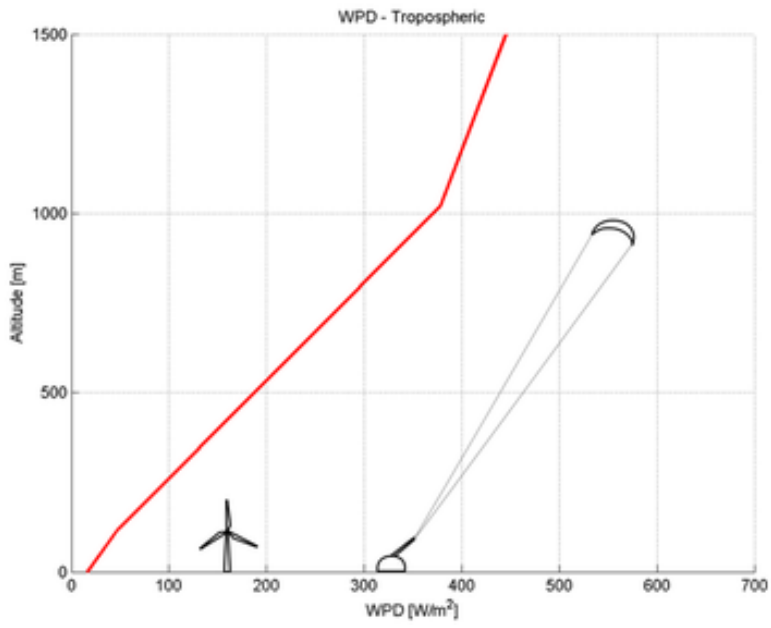

Figure 1 Wind velocity and density at various height [3] 
The new technology of kite energy harvesting offers an excellent opportunity as well as presenting difficult challenges. Even though a variety of realistic projects are being worked on, the lack of published material and publically available simulations poses difficulties in the current investigation. Firstly, we will explore the character of different types of kites, review the kite motion in the air in order to build a basic computer model for the chosen types of kite for further advanced development.

\section{SELECTION OF KITE}

Different kites have different interactions with the wind and various mechanical motions. A kite that can produce an efficient upward thrust while at the same time remains simple structure would be ideal. Other factors for consideration are price, availability, shape, mass, and surface area. A variety of kites were considered for selection

\section{A. Diamond Shape Kite}

As a classic design that has been proven to be popular throughout the years, a kite in a diamond shape is an excellent choice. With predictable upward lift and drag based upon a single flat surface plane with minimal thickness, it is straightforward to model the interaction between this kite and the wind vector. Costs of design and building of the kite are small. The mass can also be kept minimal by using metal bars as frames and nylon as the surface. However, the gain in the lift of the kite per increased surface area is not as efficient as other kites. Also, they are relatively uncontrollable for power generation purposes and the wind speed operation range is comparatively narrower.

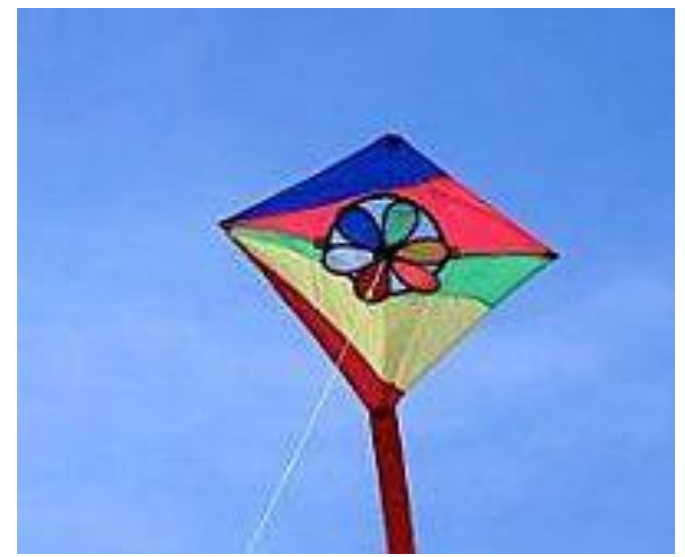

Figure 2 Diamond shape kite

\section{B. Barn Door Kite}

The barn door kite is a large, pentagon-shaped kite with sides elongated to form a stout body for flight. This kite has a slight curvature towards the centre of the original pentagon shape where all the frames cross over. Giving it a larger, tortoise shell-like appearance, this protrusion mimics the behaviour of an airplane wing where the pressure difference created is larger, thereby giving it bigger lift compared to the diamond kite. The reinforced frames are required to keep the kite from ripping from being ripped. The cost is slightly higher than for the diamond kite. The mass is also within an acceptable standard as the lift counters the increased weight. However, the increased mass means a higher wind speed is required to initially start the kite, coupled with the growing complication of five-point joints to control the kite predictably.

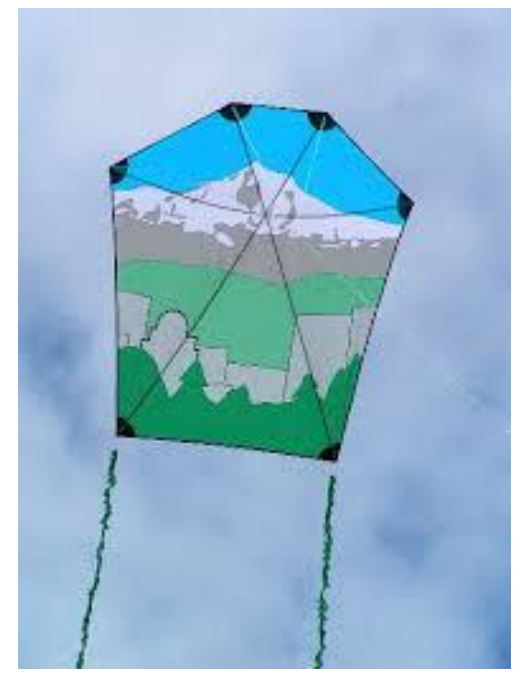

\section{Figure 3 Barn Door Kite}

\section{Surf Kite}

This kite is unique in that it contains no frames. It is essentially a floating canvas tied to two strings. This type of kite can be controlled by two lines, whereas the single line kites above are highly uncontrollable for the flight trajectory. With two-line control, the operator can steer the kite by pulling on one line to create a roll angle; the angle will then cause the kite to yaw toward to the pulling side. Meanwhile, the two-line control of power kite can generate a crosswind motion by altering the flight trajectory moving towards to the non-parallel normal wind's direction. This crosswind component increases the apparent wind on the kite.

Due to the material and the form of the kite shape, surf kites can generate more lift force and less drag force than other types of kites. This means that the kite can glide in the air for longer periods of time and can travel further to the edge of the wind window. Furthermore, this arc shape power kite can be quickly re-launched from the ground by pulling on one of the tethering lines to turn on the kite wingtip. Thus, the power kite was chosen as the kite model to be used. 


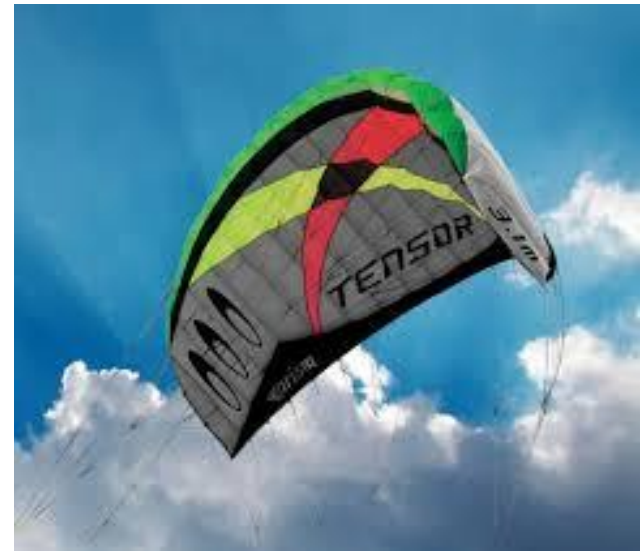

Figure 4 Surf kite

\section{SYSTEM DESCRIPTION}

\section{A. Overall system operation}

The overall system will have five key parts: aerodynamic part, kite body, mechanical part, electric machines and control system. Within the aerodynamic part, the wind force, gravity and air pressure will be calculated and translated into the same coordinate system as the kite and then act as a direct force to move the kite. The mechanical part will have three parts, which are two control tether cables (left and right) wound around two drums; there will be a drum brake coupled to the drum. Each of the drums will physically connect to the end shaft of the electric machines, which are two DC motors/generators. In the end, there will be a control system controlling the motor operation and the drum brake. The detailed operation process is illustrated below. In this case, kite operation will have three phases, which are known as traction phase, cross-feed traction phase and retraction phase. These are explained respectively according to the diagram below:

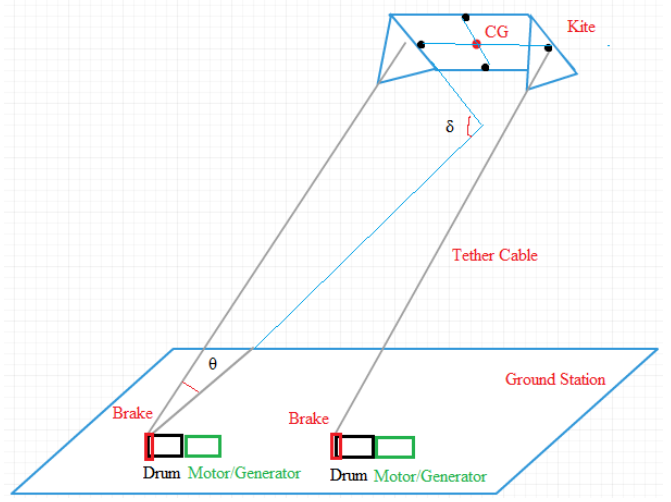

Figure 5 Overall kite system

\section{B. Traction Phase}

In this paper, the kite will be modelled as rigid body subject only to gravity and wind forces. Then another four points are distributed equally from the center gravity $(\mathrm{CG})$ so that the kite can be treated as a plane with an area of S. The kite used in this project has a wingspan distance of two meters. The wind direction (wind tunnel test condition) here is defined as always perpendicular to the kite surface, and the kite will move along with the angle of $\delta$. The tether cable will be treated as two rigid body lines attached to the left end of the kite and the right end of the kite. The tether cable and the referenced ground will have angle $\theta$.

After the kite has launched, a detailed treatment of the launching process is not within the scope of this paper; the kite will be in the initial traction phase. Which means the kite will pull both tether lines, hence rotating both generator shafts to produce power at the same time until one side of the cable length reaches the preset values. In this case, the left cable will be set to the initial conditions, therefore, when left cable (L) reaches the preset value, the drum can be stopped by applying the drum brake force. Thus, the left generator will stop. The right generator will go further for some time (distance) until the right cable $(\mathrm{R})$ reaches the preset distance. The right drum brake will then be applied to the drum to stop rotating.

Hence, the different length of these two ropes will result in the angle of $\Psi$ as seen in figure 16 in the horizontal plane (YZ plane). Due to this angle, the kite position is no longer in an equilibrium position; it will move sideways due to the normal component of the wind force. At the same time, while the kite is moving sideways, the left side of the rope is released so that it is equal length to the right side of the rope. Therefore, the kite will reach another equilibrium position in a different location. The kite will then locate on the left-hand side of the original track.

The angle $\Psi$ determines how far a kite will fly horizontally before it starts to fall. If this angle is too small, it will not generate the sufficient displacement, or if the angle is too big, it will result in the kite tipping over. The angle should be ideally to be 30 degrees.

Therefore by applying the simple math, for a kite with a wingspan of two meters, to create an optimal 30 degrees of $\Psi$, $\sin ^{-1} \Delta \mathrm{L} / d=30$; then, $\Delta \mathrm{L}=\mathrm{L}_{\mathrm{L}}-\mathrm{L}_{\mathrm{R}}=1 \mathrm{~m}$. Figure 6 a shows the initial traction phase of the kite, in which the left side and right side of the rope will be pulled at the same time.

Figure $6 \mathrm{~b}$ shows when the left side of the rope reaches 20 meters, the drum brake will clamp on to stop and right side of the rope will continue to go for further 1 meter to create the angle $\Psi$, which result in the kite flight toward to the left side, at the same time, release the left side of rope so that both ropes have the same length of 21 meters, the kite aerodynamic will then adjust by itself to find another equilibrium in a new displacement. 


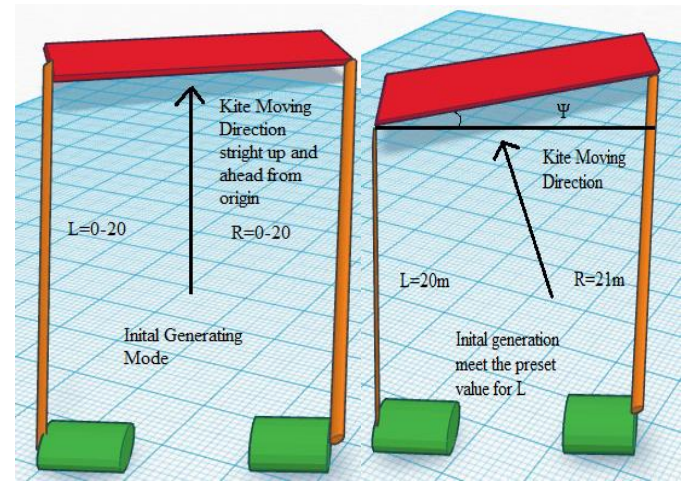

Figure 6 a) Initial traction phase b) Traction phase

\section{Cross-Feed Traction Phase}

By allowing the left-hand side of the rope to pull (generation mode) for 1 meter while the right-hand side drum is being held, the kite then will move to the right-hand side. Once the left-hand side of the rope reaches 22 meters, the drum brake will be applied on the drum again. Meanwhile, the righthand side of the drum brake is freed until rope $\mathrm{R}$ reaches the same length as the left-hand side of the rope, which is 22 meters. The kite will then move to the right-hand side from the original track.

After the kite arrival at the equilibrium position on the right-hand side, the right-hand side of the drum brake will be released again so the right-hand side of the rope will pull for another 1 meter. Due to the component of the wind, the kite will move towards to left-hand side again, while the left-hand side of the brake is released for 1 meter so that it will be equal to the right-hand side rope length which is 22 meters. The kite will reach a new equilibrium position.

\section{Retraction Phase Cross}

The cross-feed traction phase will repeat until the left-hand side of the rope length first reaches the maximum, in this case, 40 meters. Only this time, the right-hand side of the brake will not release the right hand of the rope; the kite can no longer form an equilibrium position and will start to fall with an angle $\Psi$. The retraction phase will begin to act; the same two DC generators now will behave like a motor to wind back the rope at the same speed.

The $\Psi$ angle will ensure that the tension force exerted by the kite aerodynamic on the rope will be at the minimum. (low lift force and low drag force). In this phase, the motor will need to use as little power as possible to retract the kite and work as fast as possible to prevent the kite falling speed from being quicker than the retraction speed. At the same time, both motors need to work at the same speed and time.

When the left-hand side of the rope reduces to 5 meters again, both sides of the motor will stop working and transfer to generation mode again. The left-hand side of the drum brake will be applied to the left-hand side of the drum, and release the right-hand side of the drum for another 1 meter so that $\mathrm{L}=\mathrm{R}=5$ meters and the kite can be balanced out at its equilibrium state. At this stage, the operation of the generator is repeated and so on. Figure 7 below illustrates one complete cycle of the traction and retraction phase:

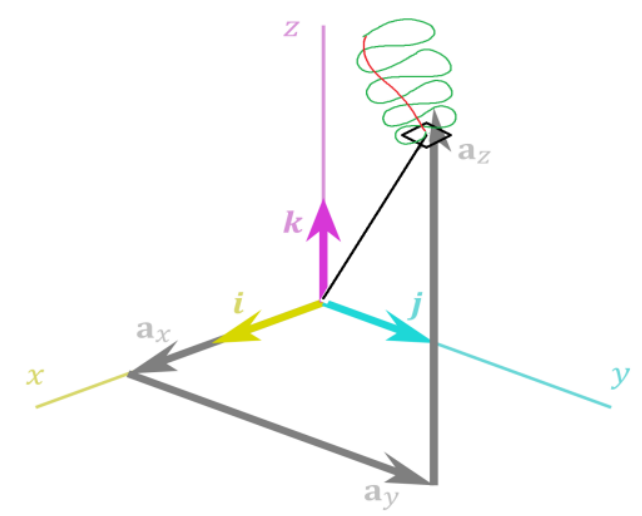

Figure 7 Flight trajectory

\section{RESULTS}

Figure 8 below illustrates the altitude and $\mathrm{X}$ direction of the kite position concerning time. During the traction phase, due to the aerodynamic force, the kite will climb and move towards the downwind. The trap cut indicates that the cable is doing the cross-feed until the cable reaches maximum length, then the retraction phase will start at the 30 s. The graph shows that the kite rapidly moves upwind until it reaches a quasi-equilibrium state, then the next traction phase will start again.

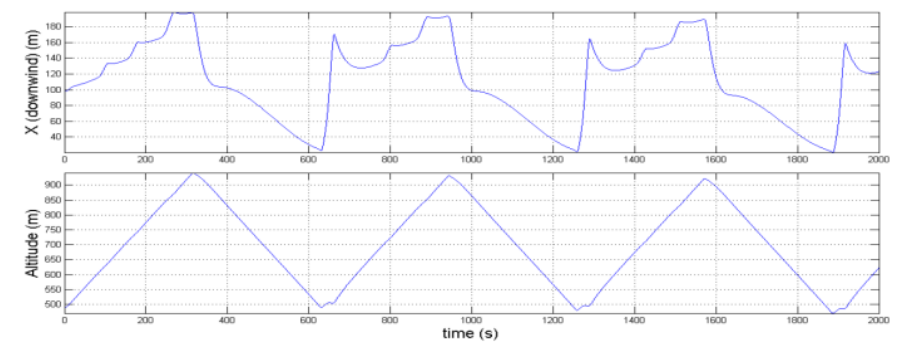

Figure 8 Kite position

The power can be calculated out by using mathematic formula $P=T^{*} \omega_{m}, \omega_{m}=d \theta_{m} / d t$ [4]. Since there is no gear ratio in between (ignore the drum radius in the test), the angular displacement of the generator rotor shaft $\theta_{\mathrm{m}}$ is directly proportional to the cable speed. Therefore, the power can be presented as:

\section{Power $=$ Tension $*$ Cable Speed}

Figure 9 shows the average power generation over the simulation time is approximately $100 \mathrm{~W}$. 


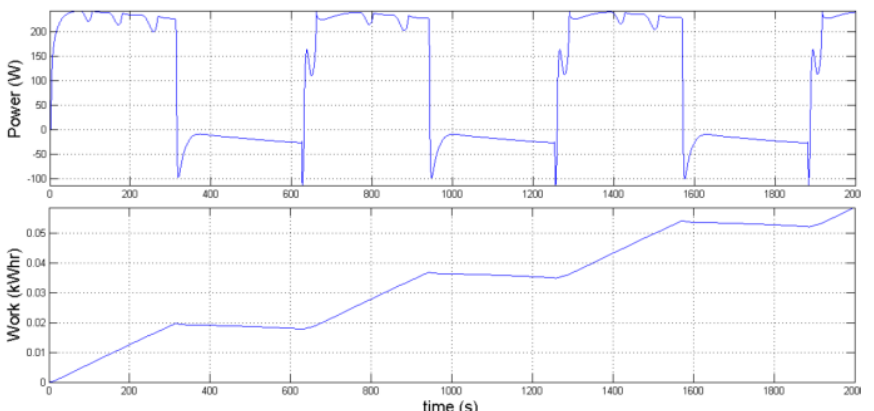

Figure 9 Power generation

For the first 300s, it is a traction phase; the power that has been generated is around $200 \mathrm{~W}$. The cupped shape at the $80 \mathrm{~s}$, 160 s and 240s are when the kite been having been steering to a different location. The retraction phase starts at 320s; the electric machine now acts as a motor; it consumes approximately $100 \mathrm{~W}$ to retrace the kite back to the original position. This result suggests that it is possible to generate power by the current system model.

\section{REFERENCES}

[1] U.S. Energy Information Administration, International Energy Statistics, $\quad$ Retrieved from http://www.eia.gov/cfapps/ipdbproject/iedindex3.cfm?tid=2\&pid=2\&aid =2\&cid=ww, \&syid=1980\&eyid=2012\&unit=BKWH $\quad$ (accessed February 1, 2016)

[2] Wind-power Program, The Betz limit. Retrieved from http://www.windpower-program.com/betz.htm (accessed May 24, 2016)

[3] Antonello Cherubini, "Kite Dynamics and Wind Energy Harvesting," Airborne wind energy, Retrieved from http://www.antonellocherubini.com/why-airborne-wind-energy.html (accessed February 5, 2017)

[4] Loyd ML, Crosswind kite power. J.Energy 1980;4:106-11. 